\title{
EFEITO DA TAXA DE CARREGAMENTO SOBRE A REMOÇÃO DE POLUENTES DE ESGOTO EM SISTEMAS ALAGADOS CONSTRUÚDOS
}

\section{ANTONIO T. DE MATOS ${ }^{1}$, MOZART DA S. BRASIL ${ }^{2}$, PAOLA A. V. LO MONACO ${ }^{3}$}

RESUMO: objetivou-se estudar o efeito da taxa de carregamento sobre a eficiência de sistemas alagados, construídos de escoamento horizontal subsuperficial (SAC-EHSS), em remover poluentes de esgoto sanitário. Os SAC-EHSS foram alimentados com efluente proveniente de tanque séptico, sob taxas de carregamento orgânico volumétrico $\left(\mathrm{TCO}_{\mathrm{V}}\right)$ de 53 a $231 \mathrm{~g} \mathrm{~m}^{-3} \mathrm{~d}^{-1}$ de demanda química de oxigênio (DQO total) e as associadas taxas de carregamento $\left(\mathrm{TC}_{\mathrm{V}}\right)$ de demanda química de oxigênio solúvel, sólidos suspensos totais (SST), nitrogênio total (N-Total) e fósforo total (P-Total). Para avaliação de desempenho dos SAC-EHSS, as mesmas variáveis foram quantificadas em seu efluente. Relação linear e positiva foi obtida entre as taxas de remoção de DQO total, DQO solúvel e SST, e as $\mathrm{TC}_{\mathrm{V}}$, nas faixas em que foram aplicadas, além de não terem sido observados efeitos negativos desta variável sobre a eficiência na remoção desses poluentes. As taxas de remoção de $\mathrm{N}$ Total e P-Total não apresentaram, entretanto, tendência de aumento com a $\mathrm{TC}_{\mathrm{V}}$ aplicada nos SAC-EHSS, cultivados com taboa e utilizados no tratamento de efluente sanitário. As cargas aplicadas desses nutrientes devem ser consideradas referenciais no dimensionamento desses sistemas, caso se queira maximizar a remoção global de poluentes da água residuária.

PALAVRAS-CHAVE: carga orgânica, sistemas wetland, tratamento biológico.

\section{EFFECT OF LOADING RATE ON REMOVAL OF POLLUTANTS FROM WASTEWATER IN CONSTRUCTED WETLANDS}

\begin{abstract}
This research aimed to study the effect of loading rate on the efficiency of constructed wetlands with horizontal subsurface flow (CW-HSSF) to remove pollutants from wastewater. The CW-HSSF were fed with effluent from septic tank under volumetric organic loading rate $\left(\mathrm{OLR}_{\mathrm{V}}\right)$ of 53 to $231 \mathrm{~g} \mathrm{~m}^{-3} \mathrm{~d}^{-1}$ of chemical oxygen demand (total-COD) and associated loading rates $\left(\mathrm{LR}_{\mathrm{V}}\right)$ of soluble chemical oxygen demand (soluble $\mathrm{COD}$ ), total suspended solids (TSS), total nitrogen (total-N) and total phosphorus (total-P). To evaluate the performance of CWHSSF the same variables were quantified in its effluent. Linear and positive relationship was found between the removal rates of total COD, soluble COD and TSS and the $\mathrm{LR}_{\mathrm{V}}$, within the application range, and no negative effects on this variable in removal efficiencies of these pollutants. The removal rate of total-N and total-P did not show, however, tendency to increase with the $\mathrm{LR}_{\mathrm{V}}$ applied in the CW-HSSF planted with cattail and used in treating sanitary sewage. The loads applied of these nutrients must be considered referential in sizing these systems, if desired to maximize the overall removal of wastewater pollutants.
\end{abstract}

KEYWORDS: organic load, wetland systems, biological treatment.

\section{INTRODUÇÃO}

Dentre as diversas soluções propostas para o tratamento de águas residuárias domésticas, distingue-se sua disposição em Sistemas Alagados Construídos (SAC), cuja técnica tem sido utilizada em larga escala, especialmente para tratamento de esgoto sanitário, em países do

\footnotetext{
${ }^{1}$ Eng $^{\mathrm{o}}$ Agrícola, Doutor em Solos e Nutrição de Plantas, Professor do Departamento de Engenharia Agrícola, Universidade Federal de Viçosa, Viçosa - MG, atmatos@ufv.br.

${ }^{2}$ Eng ${ }^{\mathrm{o}}$ Agrônomo, Doutor em Engenharia Agrícola, Professor do Instituto Federal do Rio Grande do Norte, Natal - RN, mozart-brasil@bol.com.br.

${ }^{3}$ Eng ${ }^{a}$ Agrícola, Doutora em Engenharia Agrícola, Professora do Instituto Federal do Espírito Santo, Santa Teresa - ES, paolalm@ifes.edu.br.

Recebido pelo Conselho Editorial em: 4-11-2011
}

Aprovado pelo Conselho Editorial em: 13-10-2012

Eng. Agríc., Jaboticabal, v.33, n.2, p.319-331, març./abr. 2013 
Hemisfério Norte. No entanto, o tratamento de águas residuárias em SAC pode ser, também, considerado bastante útil em países em desenvolvimento, uma vez que se utiliza de tecnologia simples, envolvendo baixos custos de operação e manutenção (BELMONT et al., 2006; KONNERUP et al., 2009), além de ser esteticamente adequado ao ambiente. Tais aspectos tornam este tipo de tratamento ideal para aplicação em regiões carentes de saneamento básico, adequando-se perfeitamente a países de clima tropical, que possuem áreas disponíveis à sua implantação, como é o caso do Brasil (BRASIL \& MATOS, 2008).

$\mathrm{O}$ tratamento de águas residuárias sem SAC vem-se mostrando eficiente na remoção de matéria orgânica (DBO), sólidos suspensos totais (SST), nitrogênio e fósforo de efluente bruto ou primário de esgoto doméstico (ZHANG et al., 2007; CALIJURI et al., 2009; ZURITA et al., 2009; GHOSH \& GOPAL, 2010), além de coliformes (BRASIL et al., 2005; CALIJURI et al., 2009; ZURITA et al., 2009). Os estudos referentes à utilização desta tecnologia de tratamento de águas residuárias em regiões tropicais têm, entretanto, se limitado, basicamente, à avaliação da eficiência na remoção de poluentes, existindo pouca informação sobre aspectos operacionais do sistema.

Sabe-se que a eficiência na remoção de poluentes em sistemas alagados, construídos de escoamento horizontal subsuperficial (SAC-EHSS), depende da taxa de carregamento orgânico (TCO) e, como consequência, do tempo de detenção da água residuária (TUNÇSIPER et al., 2004; GHOSH \& GOPAL, 2010). Existe uma carga-limite para que o sistema possa operar com máxima eficiência, e a velocidade de escoamento da água residuária no espaço poroso está diretamente associada ao tempo de contato do material orgânico em suspensão na água residuária e o biofilme, desenvolvido aderido ao meio suporte e ao sistema radicular da vegetação em cultivo no SAC-EHSS.

A USEPA (2000) sugere que as taxas de carregamento orgânico superficial $\left(\mathrm{TCO}_{\mathrm{S}}\right)$ em SACs sejam de 40 a $80 \mathrm{~kg} \mathrm{ha}^{-1} \mathrm{~d}^{-1}$ de DBO. Referindo-se ao Tempo de Detenção Hidráulica (TDH), KADLEC \& WALACE (2009) sugerem entre 4 e 7 dias. Apesar disso, tendo em vista a necessidade de redução da área necessária e os custos de construção dos SAC-EHSS, tem sido avaliadas TCOs maiores ou TDH, menores, notadamente para condições climáticas de países de clima tropical. MATOS et al. (2010a), aplicando TCO Te 66 a $571 \mathrm{~kg} \mathrm{ha}^{-1} \mathrm{~d}^{-1}$ (TCOv de 50 a $433 \mathrm{~g} \mathrm{~m}^{-3} \mathrm{~d}^{-1}$ ) de esgoto sanitário, com TDH fixo de 4,8 d, obtiveram remoções de 78,5 a $96 \%$ na DBO e de 85 a $97 \%$ na DQO. Os mesmos autores verificaram que a eficiência na remoção de poluentes em SAC-EHSS tende a aumentar com o aumento na taxa de aplicação, até ser alcançado um máximo, a partir do qual passa a ocorrer decréscimo na eficiência do sistema, corroborando o modelo cinético de reação saturada para elevados aportes orgânicos.

Com base na cinética de reação de $1^{\mathrm{a}}$ ordem, pressupõe-se que a degradação do material orgânico ocorra linearmente com a concentração, não existindo limites para que ocorra degradação. $\mathrm{Na}$ concepção de degradação de zero ordem, pressupõe-se que exista um limite de ocupação dos sítios enzimáticos dos microrganismos degradadores e, a partir dele, não haja mais relação linear entre a degradação e as cargas aportadas ao sistema. Assim, enquanto esses sítios não são totalmente ocupados, ocorre degradação de primeira ordem; posteriormente, com o aumento da concentração orgânica, a degradação passa a ser de ordem zero e não aumentará proporcionalmente aos aportes. Considerando que os SAC-EHSS são reatores com meio suporte, apresentando espaço poroso limitado, estará passível da ocorrência de degradação de ordem zero, no caso de aportes orgânicos excessivos.

Como os estudos sobre técnicas de tratamento de águas residuárias em sistemas alagados, construídos de escoamento horizontal subsuperficial (SAC-EHSS), são relativamente recentes, principalmente em regiões de clima tropical, neste trabalho teve-se por objetivo estudar o efeito da taxa de carregamento orgânico e as associadas taxas de carregamento de outros poluentes sobre a eficiência de remoção de poluentes de esgoto sanitário. 


\section{MATERIAL E MÉTODOS}

O experimento foi conduzido na Área Experimental de Tratamento de Resíduos Urbanos do Departamento de Engenharia Agrícola, Universidade Federal de Viçosa, em Viçosa - MG. Quatro leitos de sistemas alagados, construídos de escoamento horizontal subsuperficial (SAC-EHSS), foram construídos, em paralelo (Figura 1), nas dimensões de $24 \mathrm{~m}$ de comprimento, 1,0 m de largura e profundidade inicial de $0,35 \mathrm{~m}$ de leito-suporte, com declividades de $0,5 \%, 1,0 \%$ e $1,5 \%$, e impermeabilizados com geomembrana de policloreto de vinila (PVC), com espessura de 0,50 $\mathrm{mm}$, para tratamento secundário/terciário de esgoto sanitário doméstico (Figura 2).

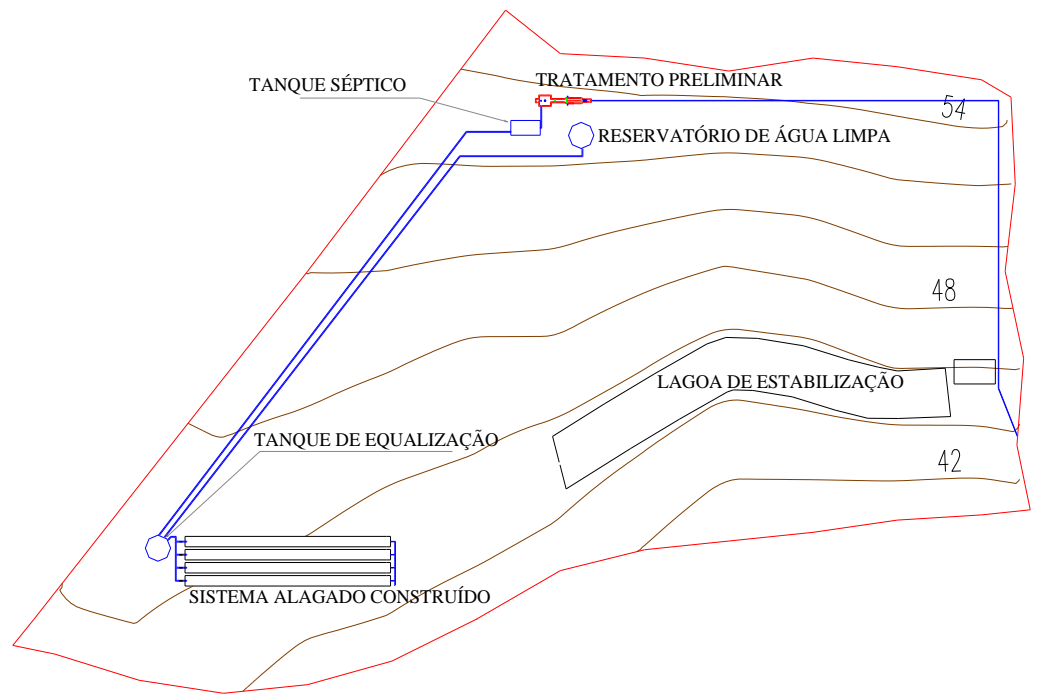

FIGURA 1. Planta da situação das unidades experimentais na Área Experimental de Tratamento de Resíduos Urbanos, do DEA/UFV. Plant situation of experimental units at the Experimental Treatment of Urban Waste, DEA/UFV.
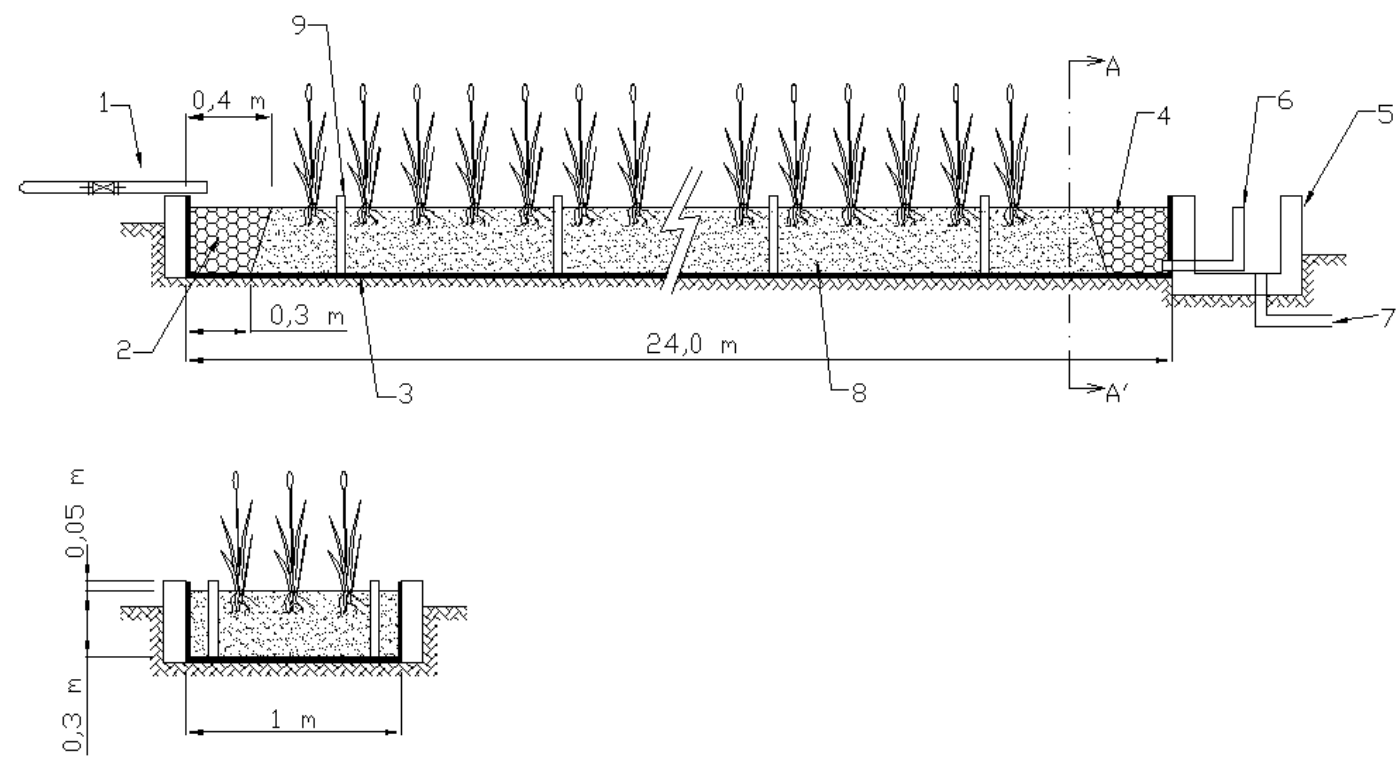

FIGURA 2. Desenho esquemático de um leito do SAC-EHSS: (1) dispositivo de distribuição de afluente; (2) zona de entrada; (3) geomembrana impermeável; (4) zona de saída; (5) caixa de coleta; (6) dispositivo de descarga e controle de nível; (7) esgotamento do efluente; (8) meio suporte (substrato) e (9) pontos internos de coleta de amostras do líquido residente. Sketch of a SAC-EHSS bed: (1) delivery device of the influent, (2) entry zone, (3) impermeable geomembrane, (4) exit zone, (5) collection box, (6) device of discharge and water level control, (7) the effluent sewage (8) support means (substrate) and (9) internal points of sampling the liquid resident. 
Como meio suporte, utilizou-se brita \# 0 (diâmetro $-\mathrm{D}_{60}=7,0 \mathrm{~mm}, \mathrm{Cu} \mathrm{D}_{60} / \mathrm{D}_{10}=1,6 \mathrm{e}$ volume de vazios de 48,4\%), sendo que, na zona de entrada, utilizou-se brita \# 1.Considerando que, em razão de os SAC-EHSS apresentarem diferentes declividades dos fundos e ter sido mantida a profundidade ao final em todos os sistemas em $0,28 \mathrm{~m}$, o volume útil nos SAC-EHSS de declividades de 0,5\% (SAC-EHSS 1), 1,0\% (SAC-EHSS 2 e 3) e 1,5\% (SAC-EHSS 4) foi, respectivamente, de $5,568 \mathrm{~m}^{3}, 4,404 \mathrm{~m}^{3}, 4,308 \mathrm{~m}^{3}$ e $3,262 \mathrm{~m}^{3}$. A macrófita emergente cultivada nos SAC-EHSS foi a taboa (Typha sp), com densidade de plantio de 8,5 propágulos $\mathrm{m}^{-2}$.

Os quatro SAC-EHSS foram alimentados com efluente sanitário, proveniente de tanque séptico, sob as condições operacionais apresentadas na Tabela 1.

TABELA 1. Valores médios de vazão $\left(\mathrm{Q}_{\mathrm{m}}\right)$, de tempo de detenção hidráulica (TDH), de fluxo no sentido transversal $\left(\mathrm{q}_{\mathrm{T}}\right)$ e de taxa de aplicação hidráulica [em base superficial $\left(\mathrm{q}_{\mathrm{a}}\right)$ e em base volumétrica $\left(\mathrm{q}_{\mathrm{v}}\right)$ ] aplicados no leito dos SACs. Mean values of flow $\left(\mathbf{Q}_{\mathbf{m}}\right)$, the hydraulic detention time (HDT), transversal flux $\left(\mathbf{q}_{\mathrm{T}}\right)$ and hydraulic application [based on surface $\left(q_{a}\right)$ and on a volumetric basis $\left(q_{v}\right)$ ] applied in the bed of SACs.

\begin{tabular}{cccccc}
\hline SAC & $\begin{array}{c}Q_{m}^{*} \\
\left(\mathrm{~m}^{3} \mathrm{~d}^{-1}\right)\end{array}$ & $\begin{array}{c}T D H \\
(\mathrm{~d})\end{array}$ & $\begin{array}{c}q_{T} \\
\left(\mathrm{~m}^{3} \mathrm{~m}^{-2} \mathrm{~d}^{-1}\right)\end{array}$ & $\begin{array}{c}q_{a} \\
\left(\mathrm{~mm} \mathrm{~d}^{-1}\right)\end{array}$ & $\begin{array}{c}q_{v} \\
\left(\mathrm{~m}^{3} \mathrm{~m}^{-3} \mathrm{~d}^{-1}\right)\end{array}$ \\
\hline 1 & 1,437 & 1,9 & 6,19 & 59,8 & 0,533 \\
2 & 1,135 & 1,9 & 6,19 & 47,3 & 0,533 \\
3 & 0,556 & 3,8 & 3,10 & 23,2 & 0,258 \\
4 & 0,840 & 1,9 & 6,19 & 35,0 & 0,533 \\
\hline
\end{tabular}

* valor médio entre a vazão afluente e efluente

As taxas de aplicação hidráulica $(q)$ foram mantidas constantes, ao longo de todo o experimento, tendo sido estabelecidas para que se obtivessem dois tempos de detenção hidráulica (TDH): de 1,9 dia, nos SAC-EHSS 1; 2 e 4, e de 3,8 dias, no SAC 3. Entretanto, para se obterem $\mathrm{TCO}_{\mathrm{V}}$ crescentes em três fases sucessivas nos SAC-EHSS, foram feitas diluições do efluente de tanque séptico, utilizando água "bruta" (coletada em manancial superficial), nas seguintes proporções e períodos de aplicação:

- $1^{\text {a }}$ Fase: $50 \%$ de efluente do tanque séptico + 50\% de água bruta (14/07 a 07/10/2004);

- $2^{\text {a }}$ Fase: $75 \%$ de efluente do tanque séptico + 25\% de água bruta (08/10 a 09/12/2004); e

- $3^{\text {a }}$ Fase: $100 \%$ de efluente do tanque séptico (10/12/2004 a 31/01/2005).

Em cada fase, foram efetuadas três amostragens para quantificação da demanda química de oxigênio total (DQO total) e solúvel (DQO solúvel), determinadas pelo método de oxidação química em refluxo aberto; sólidos suspensos totais (SST), determinados pelo método gravimétrico, com a utilização de membranas de fibra de vidro $(0,45 \mu \mathrm{m}$ de diâmetro de poro); nitrogênio total (N-Total), determinado pelo processo semimicro Kjeldahl, e fósforo total (P-Total), determinado por meio de digestão nítrico-perclórico da amostra, e quantificação em espectrofotômetro. As análises laboratoriais foram realizadas em conformidade com as recomendações do Standard Methods (APHA et al., 2005), no Laboratório de Qualidade da Água, do Departamento de Engenharia Agrícola da Universidade Federal de Viçosa.

Os resultados das análises foram utilizados para o cálculo das $\mathrm{TC}_{\mathrm{V}}$, cujas faixas de aplicação estão apresentadas na Tabela 2, e avaliação da eficiência do sistema. As taxas de carregamento volumétricas $\left(\mathrm{TCO}_{\mathrm{V}}\right.$ e $\left.\mathrm{TC}_{\mathrm{V}}\right)$ foram obtidas multiplicando-se os valores de vazões médias afluentes pela concentração do constituinte, referente a cada amostragem, dividindo, posteriormente, o resultado pelo volume de vazios do meio suporte (volume útil multiplicado pela macroporosidade) nos respectivos SAC-EHSS. 
O período de monitoramento dos sistemas foi de seis meses, sendo que, aos três meses, a biomassa aérea da taboa foi cortada a uma altura de $10 \mathrm{~cm}$ em relação à superfície do meio suporte e removida do sistema.

Os dados obtidos de remoção percentual e de taxa de remoção de poluentes foram plotados em função das $\mathrm{TC}_{\mathrm{V}}$ aplicadas e, quando os coeficientes de determinação foram maiores que 50\%, as equações foram apresentadas; caso contrário, foram apresentadas apenas as médias.

TABELA 2. Faixas de taxa de carregamento volumétrico $\left(\mathrm{TC}_{\mathrm{V}}\right)$ de DQO total, DQO solúvel, SST, N-Total e P-Total, aplicadas durante o período de avaliação e calculadas com base nos resultados de análises de três amostras, por fase de operação do sistema. Ranges of volumetric loading rate $C_{V}\left(\mathrm{~g} \mathrm{~m}^{-3} \mathrm{~d}^{-1}\right)$ of total COD, soluble COD. SST, total-N and total-P applied during the evaluation period and calculated based on the results of analysis of three samples, per phase of system operation.

\begin{tabular}{|c|c|c|c|c|c|c|c|c|c|c|c|c|c|c|c|}
\hline \multirow{3}{*}{$\begin{array}{l}\text { SAC- } \\
\text { EHSS }\end{array}$} & \multicolumn{15}{|c|}{$\mathrm{TC}_{\mathrm{V}}\left(\mathrm{g} \mathrm{m}^{-3} \mathrm{~d}^{-1}\right)$} \\
\hline & \multicolumn{3}{|c|}{ DQO } & \multicolumn{3}{|c|}{ DQO solúvel } & \multicolumn{3}{|c|}{ SST } & \multicolumn{3}{|c|}{ N-Total } & \multicolumn{3}{|c|}{ P-Total } \\
\hline & Fase 1 & Fase 2 & Fase 3 & Fase 1 & Fase 2 & Fase 3 & Fase 1 & Fase 2 & Fase 3 & Fase 1 & Fase 2 & Fase 3 & Fase 1 & Fase2 & Fase 3 \\
\hline 1 & $92-125$ & $147-189$ & $193-226$ & $42-97$ & $74-88$ & $63-99$ & & $34-48$ & $35-56$ & $8-20$ & $16-23$ & $19-22$ & $1,9-2,6$ & $3,6-4,2$ & $-5,8$ \\
\hline 2 & $91-123$ & $144-185$ & $193-226$ & $42-96$ & $73-87$ & $63-99$ & & $34-47$ & $35-56$ & $9-18$ & $9-23$ & & $1,9-2,5$ & $3,5-4,1$ & $4,6-5,8$ \\
\hline 3 & $53-72$ & $79-102$ & $102-120$ & $24-56$ & $40-48$ & $33-52$ & & $19-26$ & $18-30$ & $6-12$ & $9-12$ & $11-12$ & $1,1-1,5$ & $1,9-2,3$ & $2,5-3,0$ \\
\hline 4 & $100-135$ & 154-199 & $198-231$ & $46-105$ & $78-93$ & 64-101 & & $36-51$ & $35-58$ & $10-19$ & $18-24$ & $21-23$ & $2,1-2,8$ & $3,8-4,4$ & $4,7-5,9$ \\
\hline
\end{tabular}

Em que, SAC-EHSS 1 - declividade de 0,5\% e TDH de 1,89 d; SAC-EHSS 2 - (declividade de 1,0\% e TDH de 1,89 d; SAC-EHSS 3 - declividade de $1,0 \%$ e TDH de $3,75 \mathrm{~d}$, SAC-EHSS 4 - declividade de 1,5\% e TDH de 1,89 d.

\section{RESULTADOS E DISCUSSÃO}

$\mathrm{Na}$ Tabela 3, estão apresentados os valores de eficiência de remoção de DQO total, DQO solúvel, SST, N-total e P-total, em função das $\mathrm{TC}_{\mathrm{V}}$ aplicadas.

TABELA 3. Eficiências na remoção (\%) de DQO total, DQO solúvel, SST, N-total e P-total, nas respectivas amostragens efetuadas nos SAC-EHSS. Removal efficiencies (\%) of total COD, soluble COD, TSS, total-N and total-P in the respective samplings in the SAC-EHSS.

\begin{tabular}{|c|c|c|c|c|c|c|c|c|c|c|c|c|}
\hline \multirow{2}{*}{ Variável } & \multirow{2}{*}{ SAC } & \multicolumn{3}{|c|}{ Fase 1} & \multicolumn{3}{|c|}{ Fase 2} & \multicolumn{3}{|c|}{ Fase 3} & \multirow{2}{*}{ Média } & \multirow{2}{*}{$\begin{array}{l}\text { Desvio_ } \\
\text { Padrão }\end{array}$} \\
\hline & & Am 1* & Am 2 & Am 3 & Am 4 & Am 5 & Am 6 & Am 7 & Am 8 & Am 9 & & \\
\hline \multirow{4}{*}{ DQO total } & 1 & 81 & 85 & 87 & 87 & 87 & 86 & 87 & 89 & 83 & 86 & 2 \\
\hline & 2 & 85 & 79 & 86 & 90 & 86 & 89 & 85 & 87 & 85 & 86 & 3 \\
\hline & 3 & 90 & 85 & 90 & 94 & 90 & 93 & 89 & 93 & 91 & 90 & 3 \\
\hline & 4 & 90 & 79 & 86 & 89 & 94 & 89 & 91 & 87 & 88 & 88 & 4 \\
\hline \multirow{4}{*}{ DQO solúvel } & 1 & 76 & 84 & 93 & 79 & 88 & 83 & 72 & 78 & 78 & 81 & 6 \\
\hline & 2 & 84 & 77 & 71 & 79 & 75 & 89 & 77 & 85 & 60 & 77 & 8 \\
\hline & 3 & 90 & 84 & 78 & 86 & 92 & 93 & 87 & 87 & 73 & 85 & 6 \\
\hline & 4 & 87 & 84 & 85 & 93 & 92 & 88 & 87 & 78 & 69 & 85 & 7 \\
\hline \multirow{4}{*}{ SST } & 1 & & & & 95 & 86 & 84 & 89 & 84 & 90 & 88 & 4 \\
\hline & 2 & & & & 93 & 92 & 99 & 88 & 85 & 100 & 93 & 6 \\
\hline & 3 & & & & 100 & 87 & 94 & 90 & 74 & 99 & 91 & 10 \\
\hline & 4 & & & & 97 & 95 & 78 & 84 & 95 & 97 & 91 & 8 \\
\hline \multirow{4}{*}{ N-total } & 1 & 8 & 40 & 29 & 32 & 51 & 25 & 13 & 11 & 29 & 26 & 13 \\
\hline & 2 & 17 & 45 & 43 & 32 & 56 & 36 & 24 & 10 & 29 & 32 & 15 \\
\hline & 3 & 42 & 52 & 82 & 62 & 76 & 53 & 50 & 50 & 47 & 57 & 14 \\
\hline & 4 & 26 & 50 & 82 & 87 & 73 & -9 & 21 & 12 & 32 & 42 & 38 \\
\hline \multirow{4}{*}{ P-total } & 1 & 31 & 59 & 77 & 46 & 43 & -8 & -13 & 11 & 32 & 31 & 30 \\
\hline & 2 & 19 & 35 & 71 & 34 & 47 & 27 & 4 & 16 & 28 & 31 & 19 \\
\hline & 3 & 56 & 27 & 97 & 93 & 67 & 38 & -18 & 30 & 44 & 48 & 35 \\
\hline & 4 & 26 & 63 & 90 & 69 & 77 & 32 & -7 & 4 & 40 & 44 & 33 \\
\hline
\end{tabular}

\footnotetext{
* Am - amostra.
} 
As TCOv aplicadas foram de 53 a $231 \mathrm{~g} \mathrm{~m}^{-3} \mathrm{~d}^{-1}$ de DQO total, e as taxas de carregamento orgânico superficial $\left(\right.$ TCO $_{\mathrm{S}}$ ), de 52 a $253 \mathrm{~kg} \mathrm{ha}^{-1} \mathrm{~d}^{-1}$ de DQO total.

As elevadas remoções de DQO total obtidas (86 a 90\%) foram superiores às encontradas por CHAGAS et al. (2011), que obtiveram remoções de 63 a 72\%, em SAC-EHSS submetidos a taxas de carregamento orgânico superficial (TCOs), que variaram de 44 a $395 \mathrm{~kg} \mathrm{ha}^{-1} \mathrm{~d}^{-1}$ de $\mathrm{DBO}_{5} \mathrm{e}$ TDHs de 3,9 a 0,75 dias, e ZURITA et al. (2009), que obtiveram remoções de 75,5 e 77,1\%, em SAC-EHSS cultivados com copo-de-leite e misto (ave-do-paraíso, antúrio e agapanto), respectivamente,submetidos a $\mathrm{TCO}_{\mathrm{S}}$ de $45,6 \mathrm{~kg} \mathrm{ha}^{-1} \mathrm{~d}^{-1}$ e TDH de 4 dias, no tratamento de efluente, de tratamento primário de esgoto doméstico.

As elevadas eficiências obtidas, neste trabalho, na remoção de SST, também foram reportadas em outros trabalhos, nos quais foram tratados diferentes tipos de águas residuárias em SAC-EHSS (BRASIL et al., 2005; KONNERUP et al., 2009; ZURITA et al., 2009; MATOS et al., 2010a, 2010c). De acordo com METCALF \& EDDY (2003), os SACs são eficientes na remoção de SST em decorrência da sedimentação nos interstícios, retenção por restrição ao escoamento (filtração) e adesão aos grânulos do material-suporte (em razão da ação da força de van der Waals).

Em relação ao N-Total, o sistema foi capaz de remover um máximo de 26 a $57 \%$ do N-Total da água residuária nos SAC-EHSS avaliados, dependendo da $\mathrm{TC}_{\mathrm{V}}$ e do $\mathrm{TDH}$, valores próximos ao encontrados por ZURITA et al. (2009), que obtiveram remoções de 53,7 e 51,7\% em SACs cultivados com copo-de-leite e SACs mistos (cultivados com antúrio, agapanto e ave-do-paraíso), respectivamente, no tratamento de efluente de tratamento primário de esgoto doméstico.

As eficiências de remoção de P-total (31 a 48\%) foram semelhantes às obtidas por ZURITA et al. (2009), de 44,7 e 35\%, em SAC-EHSS cultivados com copo-de-leite e SAC-EHSS mistos (cultivados com antúrio, agapanto e ave-do-paraíso), respectivamente, no tratamento de efluente primário de esgoto doméstico.

Verificou-se a ocorrência, por uma vez para o N-total, e por quatro vezes para o P-total, de eficiências negativas de remoção. Isso ocorreu logo após o corte e A remoção da biomassa dos SAC-EHSS, período em que a planta ainda se encontra com baixa eficiência na remoção de nutrientes, podendo ocorrer a disponibilização dos nutrientes a partir da mineralização dos resíduos da cultura.

Na Figura 3, estão apresentadas as variações nas taxas de remoção volumétricas $\left(\mathrm{TR}_{\mathrm{V}},\right)$, que é a massa do poluente removida por unidade de volume e tempo, e a eficiência de sua remoção, em função da $\mathrm{TC}_{\mathrm{V}}$. 

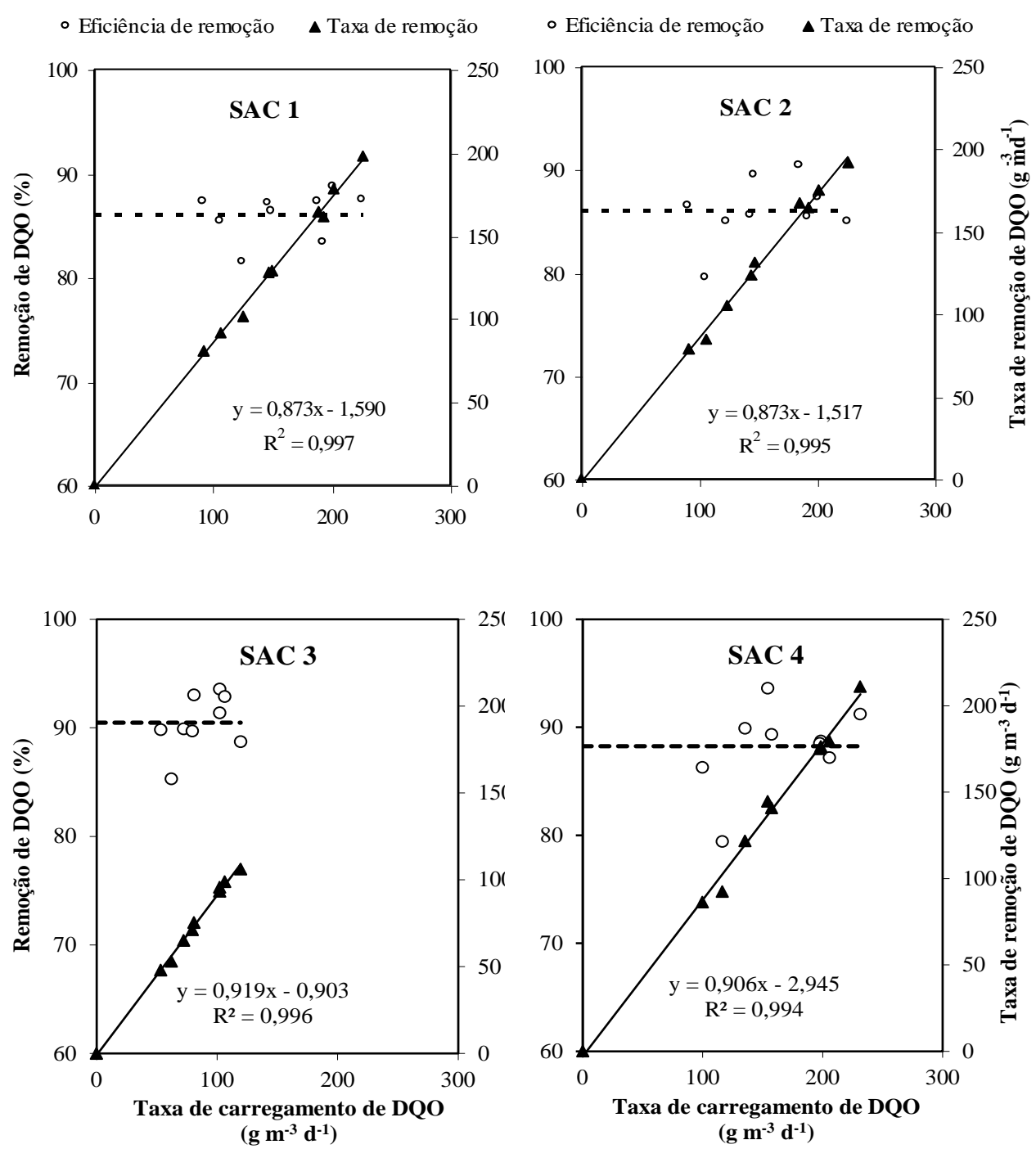

FIGURA 3. Taxas de remoção volumétrica (linha contínua) e eficiências na remoção de DQO total (linha tracejada), em função das taxas de carregamento orgânico volumétrico aplicadas. Volumetric removal rates (solid line) and removal efficiencies of total COD (dotted line) as a function of volumetric organic loading rates applied.

Elevados coeficientes de determinação $\left(\mathrm{R}^{2} \geq 0,99\right)$ foram obtidos no ajuste de equações lineares entre as $\mathrm{TR}_{\mathrm{V}}$ e as $\mathrm{TCO}_{\mathrm{V}}$ aplicadas aos quatro SACs, durante o período de avaliação do sistema, tendo as $\mathrm{TR}_{\mathrm{V}}$ de $\mathrm{DQO}$ total aumentado linearmente com a $\mathrm{TCO}_{\mathrm{V}}$. Semelhante comportamento foi reportado por TUNÇSIPER et al. (2004), embora as equações ajustadas tenham apresentado coeficientes de determinação inferiores aos obtidos no presente trabalho.

Em análise realizada, de forma isolada, do efeito das $\mathrm{TCO}_{\mathrm{V}}$ na eficiência de remoção de DQO total para cada SAC-EHSS, verificou-se não haver relação definida entre a eficiência de remoção e as $\mathrm{TCO}_{\mathrm{V}}$ aportadas, quando o TDH é mantido constante. CHAGAS et al. (2011) também não encontraram diferença na eficiência de remoção de DQO total em SAC-EHSS submetidos a diferentes TCOs, entretanto, nesse caso, o TDH decresceu proporcionalmente à TCO aplicada. A não alteração na eficiência de remoção de DQO total, observada neste trabalho, indica que a capacidade de degradação da matéria orgânica pelos microrganismos não foi superada nos SACEHSS, mesmo na maior $\mathrm{TCO}_{\mathrm{V}}$ aplicada. MATOS et al. (2010a), ao trabalharem com maiores $\mathrm{TCO}_{\mathrm{V}}$ (50 a $433 \mathrm{~g} \mathrm{~m}^{-3} \mathrm{~d}^{-1}$ ) em SACs cultivados com capim-elefante e capim-tifton 85, TDH de 4,8 d, utilizados no tratamento de águas residuárias de laticínios, verificaram que o modelo raiz quadrada foi o que proporcionou melhor ajuste para a estimativa da eficiência na remoção da DQO e DBO em função da $\mathrm{TCO}_{\mathrm{v}}$. Apenas para a estimativa de SST e para o capim-elefante é que o melhor 
ajuste obtido foi o quadrático. De acordo com os mesmos autores, as equações ajustadas indicaram que a eficiência na remoção do poluente tende a aumentar com o aumento na taxa de aplicação, até ser alcançado um máximo, a partir do qual passa a ocorrer decréscimo na eficiência de remoção.

Em relação à DQO solúvel, a $\mathrm{TR}_{\mathrm{V}}$ e a eficiência em sua remoção, em função da $\mathrm{TC}_{\mathrm{V}}$ aplicada (Figura 4), observou-se comportamento semelhante aos observados para DQO total; entretanto, houve tendência de redução na eficiência de remoção com o aumento da $\mathrm{TC}_{\mathrm{V}}$ aportada ao SAC 1 $\left(R^{2}>0,5\right)$, enquanto nos demais SACs não foi observada relação entre estas duas variáveis.
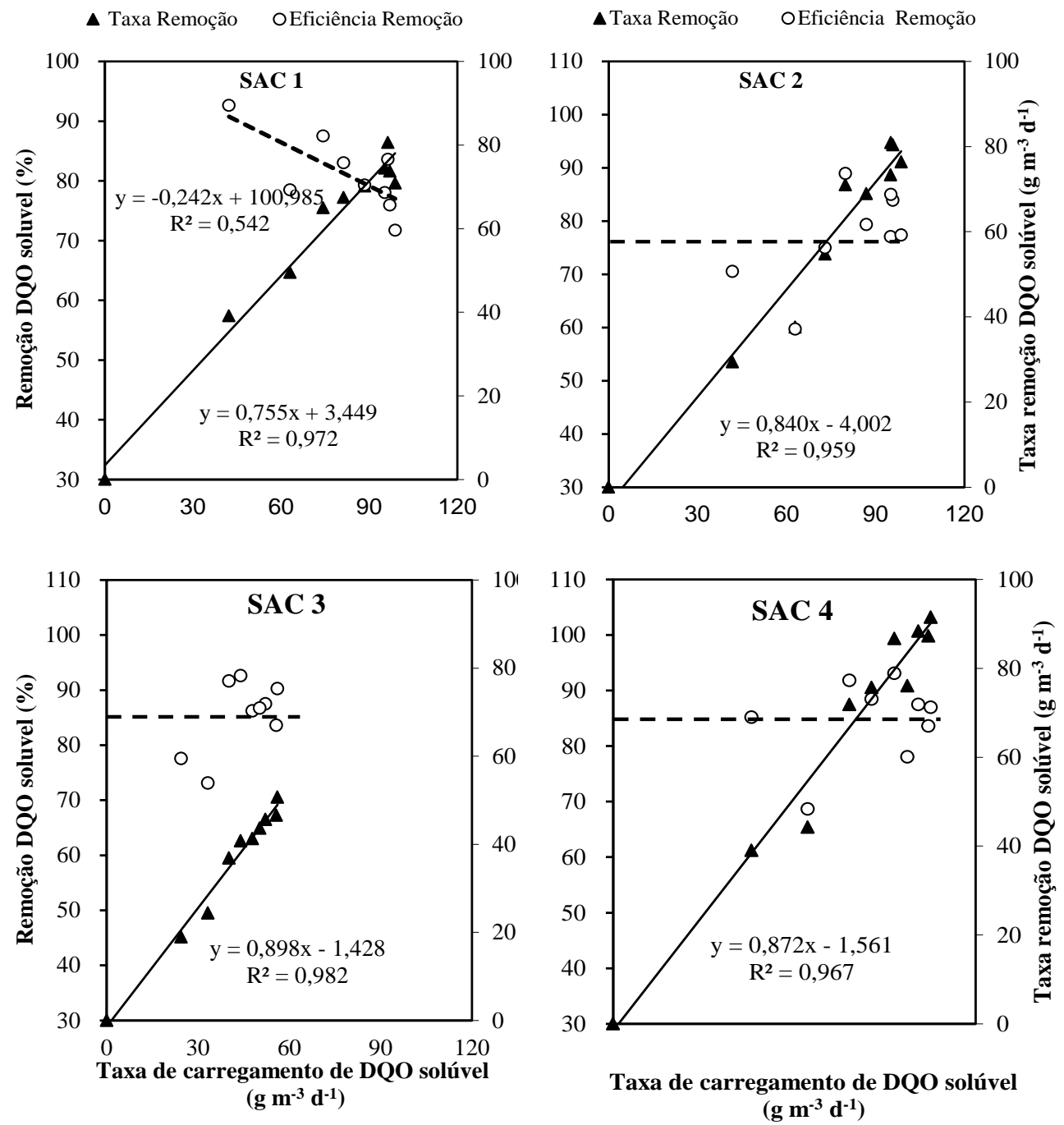

FIGURA 4. Taxas de remoção volumétrica (linha contínua) e eficiência na remoção de DQO solúvel (linha tracejada) em função das taxas de carregamento aplicadas. Volumetric removal rates (solid line) and removal efficiencies of soluble COD (dotted line) as a function of loading rates applied.

Este comportamento pode ser devido ao aparente efeito da relação área/volume, pois, no SAC 1, foi obtido o menor valor de relação área/volume e, consequentemente, a menor área superficial em relação ao volume, entre todos os SAC-EHSS. Provavelmente, isto induziu ao decréscimo na taxa de degradação da DQO solúvel, quando sua taxa de aplicação foi incrementada, o que não aconteceu com a DQO total. Essas diferenças nas remoções de DQO solúvel e total se devem ao fato de a remoção da DQO total ser mais dependente da seção transversal do meio filtrante (meio suporte) do que a DQO solúvel, pois, inicialmente, a remoção da DQO total é predominantemente física. 
Quanto à análise do efeito das $\mathrm{TC}_{\mathrm{V}}$ sobre a $\mathrm{TR}_{\mathrm{V}}$, observou-se relação linear entre essas duas variáveis, o que foi comprovado pelo bom ajuste das equações lineares $\left(R^{2} \geq 0,95\right)$. Este resultado foi semelhante àquele obtido para DQO total; entretanto, esta última apresentou coeficiente de determinação superior $\left(\mathrm{R}^{2} \geq 0,99\right)$. Apenas no SAC 1 foi observado decréscimo na eficiência de remoção de DQO solúvel com a TCv, embora neste mesmo SAC-EHSS tenha havido aumento na taxa de remoção com o aumento da TCv. No que se refere ao SST, verificou-se bom ajuste $\left(\mathrm{R}^{2} \geq 0,96\right)$ da equação linear de $\mathrm{TR}_{\mathrm{V}}$ em função da $\mathrm{TC}_{\mathrm{V}}$ (Figura 5).
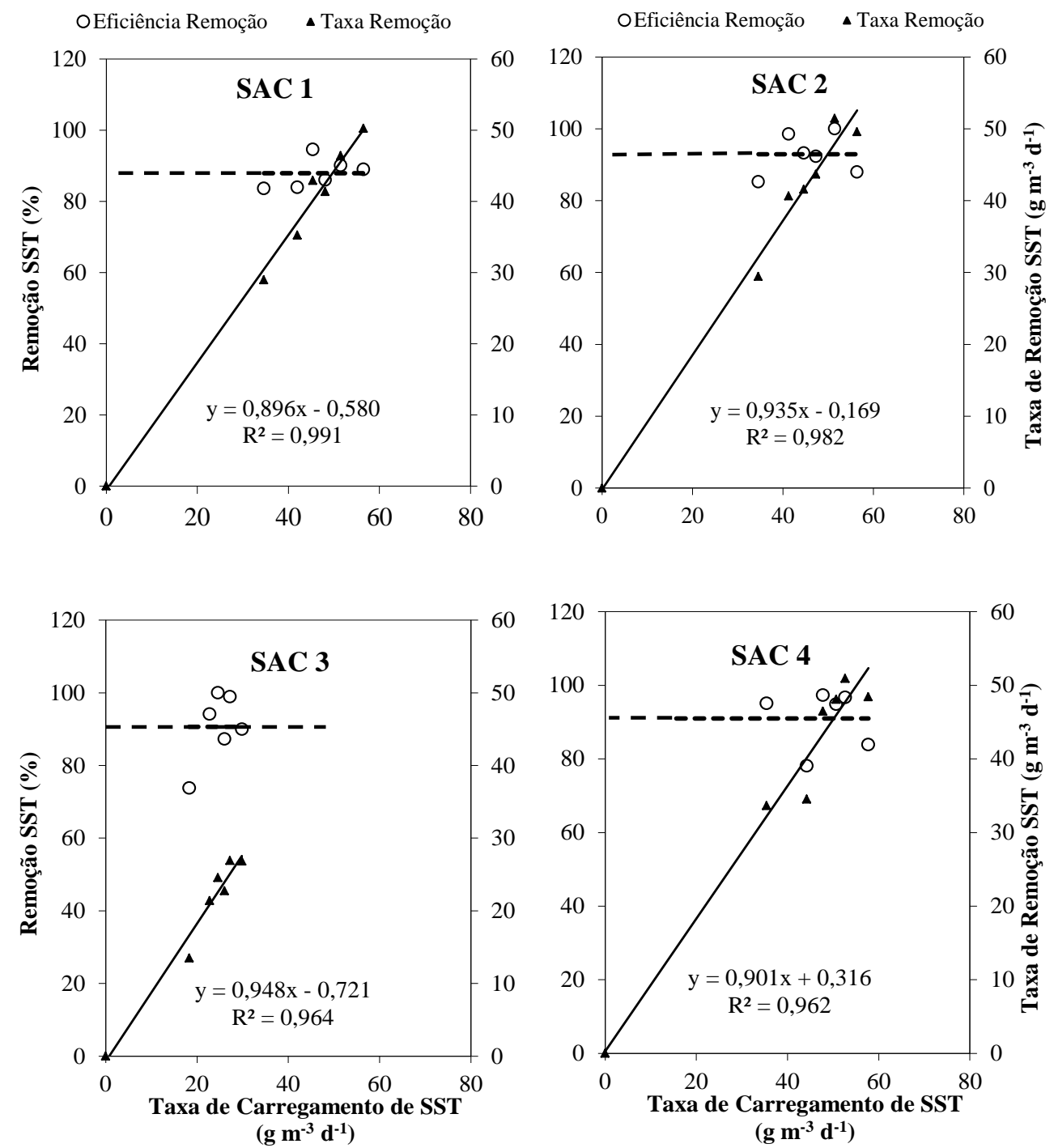

FIGURA 5. Taxas de remoção (linha contínua) e eficiências na remoção de SST (linha tracejada), em função das taxas de carregamento aplicadas. Volumetric removal rates (solid line) and removal efficiencies of SST (dotted line) as a function of loading rates applied.

Os resultados foram semelhantes aos obtidos neste trabalho para DQO total e DQO solúvel, e por TUNÇSIPER et al. (2004), para a remoção de SST, tendo sido obtido coeficientes de determinação próximos $\left(\mathrm{R}^{2} \geq 0,97\right)$.

No que se refere à relação entre $\mathrm{TC}_{V}$ e a eficiência na remoção de SST, verificou-se nenhuma influência da primeira sobre a segunda, contrariando o que foi observado por MATOS et al. (2010a), que obtiveram aumento da eficiência com a TCv, em SAC-EHSS utilizados no tratamento de águas residuárias de laticínios. 

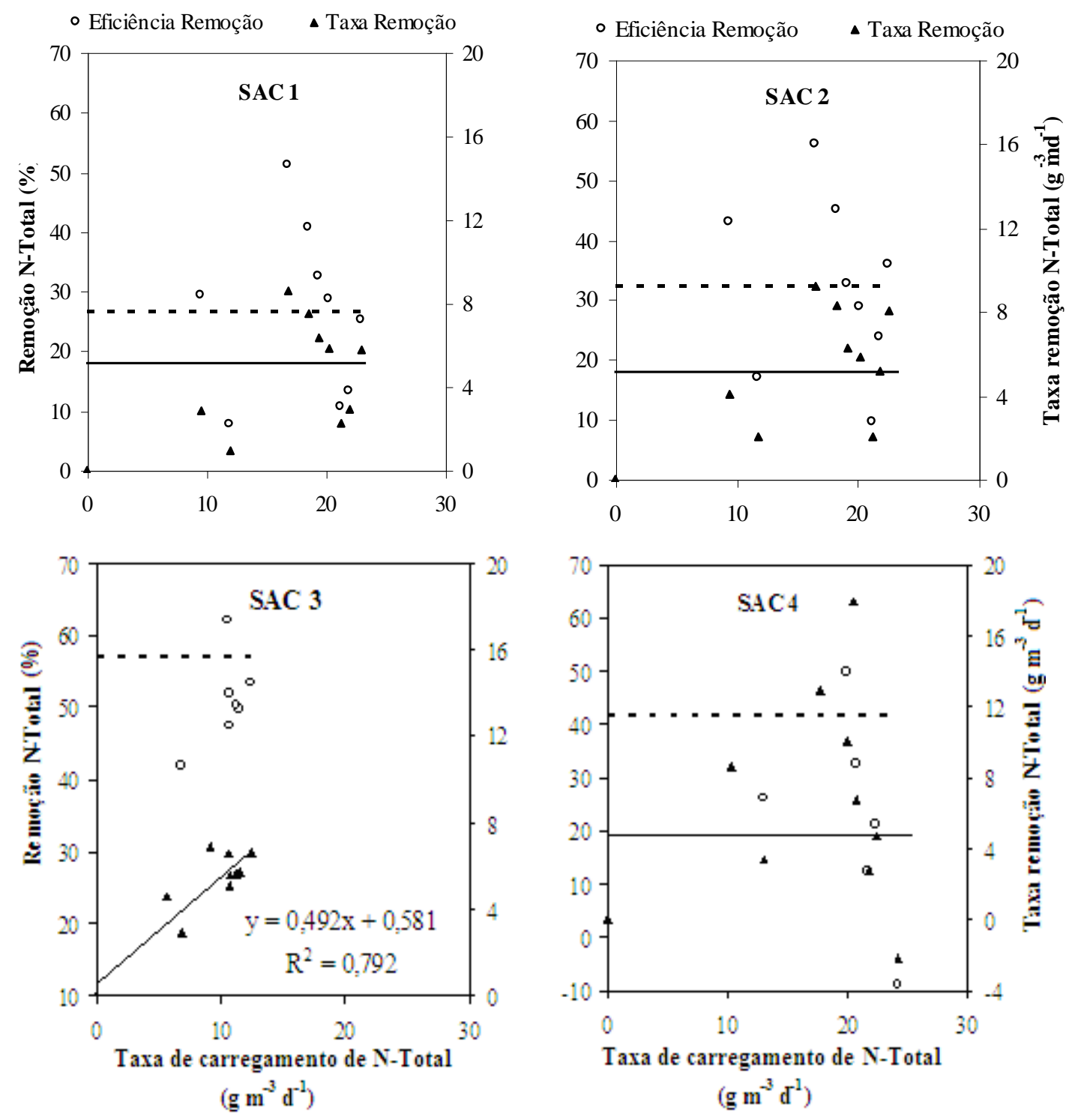

FIGURA 6. Taxas de remoção (linha contínua) e eficiências na remoção de N-Total (linha tracejada) em função das taxas de carregamento aplicadas. Volumetric removal rates (solid line) and removal efficiencies (dotted line) of total- $\mathrm{N}$ as a function of loading rates applied.

Verificou-se não haver relação definida entre as variáveis $\mathrm{TC}_{\mathrm{V}}$ (variação de 9 a $23 \mathrm{~g} \mathrm{~m}^{-3} \mathrm{~d}^{-1}$ ) e a $\mathrm{TR}_{\mathrm{V}}$ de N-Total nos SAC-EHSS 1; 2 e 4 (Figura 6). No SAC-EHSS 3, verificou-se razoável relação linear positiva $\left(R^{2}=0,79\right)$ entre essas variáveis. No entanto, neste SAC-EHSS, a TC $\mathrm{V}$ de NTotal ficou em torno da metade $\left(7 \mathrm{e} 13 \mathrm{~g} \mathrm{~m}^{-3} \mathrm{~d}^{-1}\right)$ do valor aportado nos demais SAC-EHSS. Resultados semelhantes aos obtidos no SAC-EHSS 3 foram relatados por TUNÇSIPER et al. (2004), que trabalharam com $\mathrm{TC}_{\mathrm{S}}$ de $\mathrm{N}$-Total máxima de $12 \mathrm{~kg} \mathrm{ha}^{-1} \mathrm{~d}^{-1}$, valor este próximo ao aplicado no SAC-EHSS 3, neste trabalho, que foi de 3 a $11 \mathrm{~kg} \mathrm{ha}^{-1} \mathrm{~d}^{-1}$. Entretanto, esses últimos autores trabalharam com $\mathrm{TC}_{\mathrm{V}}$ estimadas de 6 a $18 \mathrm{~g} \mathrm{~m}^{-3} \mathrm{~d}^{-1}$, valores ligeiramente superiores aos das $\mathrm{TC}_{\mathrm{V}}$ aportadas ao SAC-EHSS 3. Esses resultados sugerem que essas variáveis apresentam relação positiva, somente quando seus valores são mais baixos; todavia, no caso de valores mais elevados, pode haver limitações de remoção de N-Total, conforme foi observado nos SAC-EHSS submetidos às maiores $\mathrm{TC}_{\mathrm{V}}$.

MATOS et al. (2010a) encontraram tendência crescente na remoção de N-Total, em SACEHSS cultivados com capim-tifton 85 , no tratamento de água residuária de laticínios, quando aplicadas TCOv na faixa de 99 a $798 \mathrm{~g} \mathrm{~m}^{-3} \mathrm{~d}^{-1}$ de DQO. MATOS et al. (2010b), reportando-se ao 
mesmo trabalho, observaram que, mesmo tendo sido aplicada TCv de 1,4 a $12,7 \mathrm{~g} \mathrm{~m}^{-3} \mathrm{~d}^{-1}$ de nitrogênio total Kjeldahl, a concentração de nitrogênio no tecido foliar do capim tifton 85 não foi diferente, sendo, em média, de 3,5 dag $\mathrm{kg}^{-1}$. No presente trabalho, os SAC-EHSS 1; 2 e 4 foram submetidos a cargas de $\mathrm{N}$ acima das utilizadas pelos citados autores, podendo ter superada sua capacidade máxima de absorção pelas plantas. No SAC-EHSS 3, justamente o que foi submetido a menores $\mathrm{TCO}_{\mathrm{V}}$ e, consequentemente, maior TDH e menor carga de N-Total, houve relação linear positiva entre as $\mathrm{TC}_{\mathrm{V}}$ e $\mathrm{TR}_{\mathrm{V}}$. Ainda assim, mesmo neste SAC-EHSS, a eficiência na remoção de N-Total não aumentou com a TCv.

No que se refere à concentração de P-Total, não foi verificada nenhuma tendência de relação das $\mathrm{TR}_{\mathrm{V}} \mathrm{e}$ as eficiências de remoção desse nutriente com a $\mathrm{TC}_{\mathrm{V}}$ aplicadas (faixa de 1,1 a $5,9 \mathrm{~g} \mathrm{~m}^{-3} \mathrm{~d}^{-1}$ ) (Figura 7).
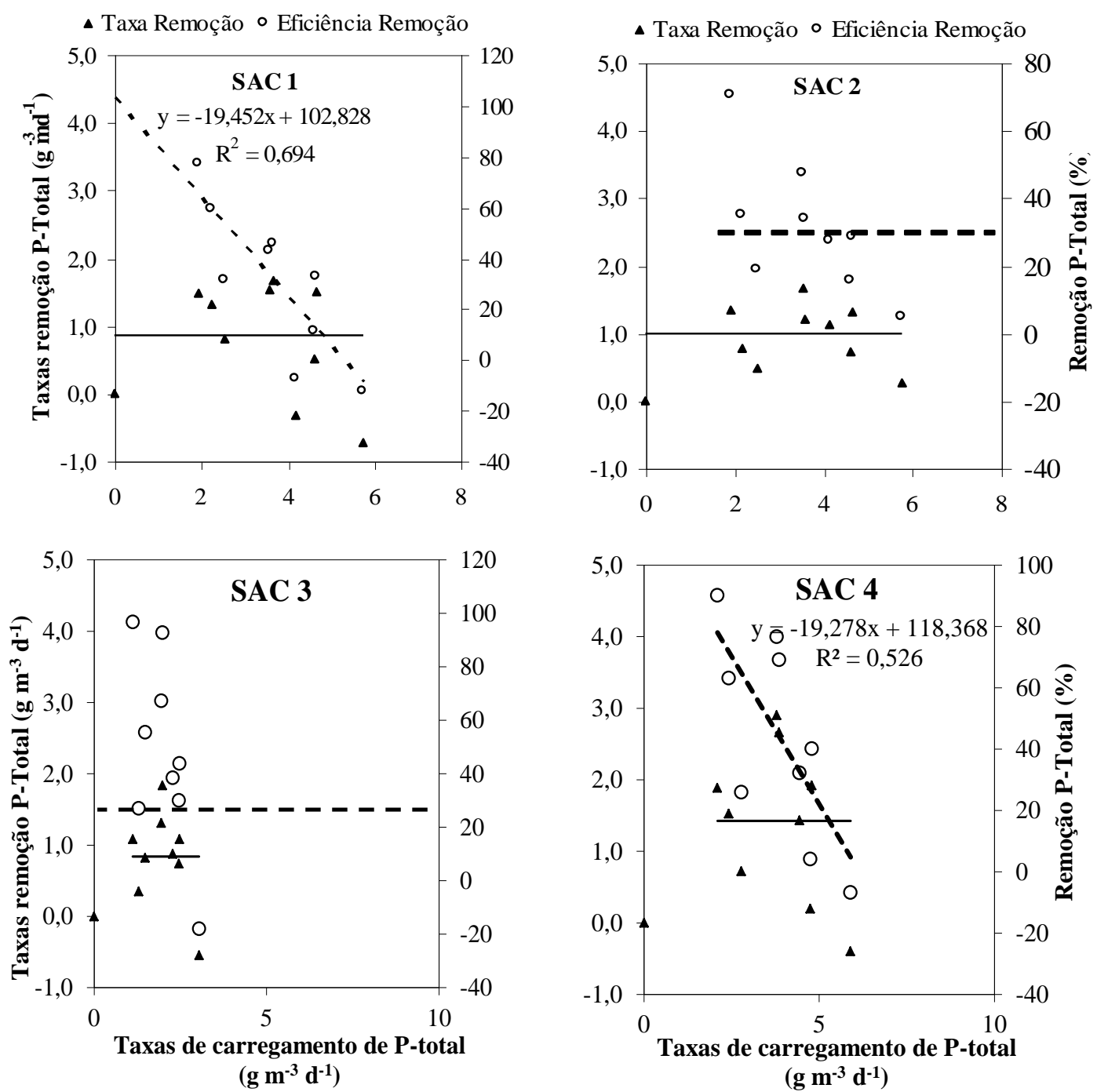

FIGURA 7. Taxas de remoção (linha contínua) e eficiências na remoção (linha tracejada) de P-Total em função das taxas de carregamento aplicadas. Volumetric removal rates (solid line) and removal efficiencies of total-P (dotted line) as a function of loading rates applied.

MATOS et al. (2010a), no tratamento de águas residuárias de laticínios em SACs com TDH fixo de 4,8 d, verificaram que, naqueles cultivados com capim-tifton 85, a remoção de P-Total foi crescente com a TCV aplicada, não sendo o mesmo comportamento observado naqueles cultivados com capim-elefante. Estes resultados são contrários aos obtidos por TUNÇSIPER et al. (2004), que apresentaram relação linear positiva $\left(\mathrm{R}^{2}=0,76\right)$ entre essas variáveis, devendo-se salientar que os 
resultados foram obtidos com TCO e taxas de aplicação hidráulicas variando concomitantemente. Quanto à relação estabelecida entre eficiência de remoção e $\mathrm{TC}_{V}$ de P-Total, verificou-se que apenas os dados gerados nos SAC-EHSS 1 e 4 proporcionaram ajuste de equações lineares, embora de inclinação negativa, apresentando coeficientes de determinação maiores ou iguais a 0,52. Os resultados indicaram que as eficiências de remoção de P-Total, em todos os SAC-EHSS, apresentaram tendência de decréscimo com a $\mathrm{TC}_{\mathrm{V}}$ aplicada, embora nos SAC-EHSS 2 e 3 não tenha sido possível o ajuste de uma equação. Com base nos resultados obtidos, entende-se que as cargas aplicadas de P-Total não devam ser superiores a $4 \mathrm{~g} \mathrm{~m}^{-3} \mathrm{~d}^{-1}$, para que não se obtenha eficiências muito baixas em sua remoção em SAC-EHSS.

\section{CONCLUSÕES}

Nas faixas de taxas de carregamento aplicadas, a remoção de DBO, DQO e SST foi crescente em SAC-EHSS cultivados com taboa, no tratamento de efluente primário de esgoto sanitário. Esses resultados indicaram ser adequada a aplicação de modelo matemático para cinética de primeira ordem no dimensionamento desses sistemas com vistas à remoção de matéria orgânica. $\mathrm{O}$ mesmo não se pode afirmar, entretanto, em relação à remoção de N-Total e P-Total, devendo estes fatores serem limitantes para a aplicação, caso se pretenda maximizar a remoção global de poluentes da água residuária.

\section{REFERÊNCIAS}

APHA - AMERICAN PUBLIC HEALTH ASSOCIATION; AWWA - AMERICAN WATER WORKS ASSOCIATION; WEF - WATER ENVIRONMENT FEDERATION. Standard methods for the examination of water and wastewater. 21.ed. Washington, 2005. $1268 \mathrm{p}$.

BELMONT, M. A.; IKONOMOU, M.; METCALFE, C. D. Presence of nonylphenolethoxylate surfactants in a watershed in central Mexico and removal from domestic sewage in a treatment wetland. Environmental Toxicology and Chemistry, New York, v.25, n.1, p.29-35, 2006.

BRASIL, M. S.; MATOS, A. T. Avaliação de aspectos hidráulicos e hidrológicos de sistemas alagados construídos de fluxo subsuperficial. Engenharia Sanitária e Ambiental, Rio de Janeiro, v.13, n.3, p.323-328, 2008.

BRASIL, M. S.; MATOS, A. T.; SOARES, A. A.; FERREIRA, P. A. Qualidade do efluente de sistemas alagados construídos, utilizados no tratamento de esgoto doméstico. Revista Brasileira de Engenharia Agrícola e Ambiental, Campina Grande, v.9, p.133-137, 2005.

CAliJURI, M. L.; BASTOS, R. K. X.; MAGAlHÃES, T. B.; CAPELETE, B. C.; DIAS, E. H. O. Tratamento de esgotos sanitários em sistemas reatores UASB/wetlands construídas de fluxo horizontal: eficiência e estabilidade de remoção de matéria orgânica, sólidos, nutrientes e coliformes. Engenharia Sanitária e Ambiental, Rio de Janeiro, v.14, n.3, p.421-430, 2009.

CHAGAS, R. C.; MATOS, A. T.; CECON, P. R.; LO MONACO, P. A. V.; FRANÇA, L. G. F. Cinética de remoção de matéria orgânica em sistemas alagados construídos cultivados com lírio-amarelo. Revista Brasileira de Engenharia Agrícola e Ambiental, Campina Grande, v.15, n.11, p.1.186-1.192, 2011.

GHOSH, D.; GOPAL, D. Effect of hydraulic retention time on the treatment of secondary effluent in a subsurface flow constructed wetland. Ecological Engineering, Amsterdam, v.36, p.1.044-1.051, 2010.

KADLEC, R.H.; WALLACE, S. D. Treatment wetlands. 2nd ed. Boca Raton: CRP Press, 2009. $1.016 \mathrm{p}$.

KONNERUP, D.; KOOTTATEP, T.; BRIX, H. Treatment of domestic wastewater in tropical, subsurface flow constructed wetlands planted with Canna and Heliconia. Ecological Engineering, Amsterdam, v.35, n.2, p.248-257, 2009. 
MATOS, A. T.; ABRAHÃO, S. S.; BORGES, A. C.; MATOS, M. P. Influência da taxa de carga orgânica no desempenho de sistemas alagados construídos cultivados com forrageiras. Engenharia Sanitária e Ambiental, Rio de Janeiro, v.15, n.2, p.83-92, 2010a.

MATOS, A. T.; ABRAHÃO, S. S.; LO MONACO, P. A. V.; SARMENTO, A. P.; MATOS, M. P. Capacidade extratora de plantas em sistemas alagados utilizados no tratamento de águas residuárias de laticínios. Revista Brasileira de Engenharia Agrícola e Ambiental, Campina Grande, v.14, n.12, p.1.311-1.317, 2010b.

MATOS, A. T.; FREITAS, W. S.; LO MONACO, P. A. V. Eficiência de sistemas alagados construídos na remoção de poluentes de águas residuárias da suinocultura. Ambi-Água, Taubaté, v.5, n.2, p.119-132, 2010c.

METCALF, E.; EDDY, H. Wastewater engineering: treatment and reuse. 4th ed. New York: MacGraw-Hill, 2003. 181 p.

TUNÇSIPER, B.; AYAZ, S. Ç.; AKÇA, L. Performances analysis and modeling of an experimental constructed wetlands. In: INTERNATIONAL CONFERENCE ON WASTE STABILIZATION PONDS; INTERNATIONAL CONFERENCE ON WETLAND SYSTEMS FOR WATER POLLUTION CONTROL, 9., 2004, Avignon. Proceedings.... CD-ROM.

USEPA. Constructed wetland treatment for municipal wastewater. Cincinnati: Office of Research and Development, 2000. 166 p. (EPA/625/r-99/010)

ZHANG, X. B.; LIU, P.; YANG, Y. S.; CHEN, W. R. Phytoremediation of urban wastewater by model wetlands with ornamental hydrophytes. The Journal of Environmental Sciences, Los Angeles, v.19, p.902-909, 2007.

ZURITA, F.; ANDA, J. D.; BELMONT, M. A. Treatment of domestic wastewater and production of commercial flowers in vertical and horizontal subsurface-flow constructed wetlands. Ecological Engineering, Amsterdam, v.35, n.5, p.861-869, 2009. 